\title{
Avaliação de informações e notificações de receitas manipuladas de drogas psicotrópicas: implicações para o uso racional de medicamentos
}

\author{
Evaluation of information and notifications of manipulated prescriptions for psychotropic \\ drugs: implications for the rational use of medicines
}
Evaluación de información y notificaciones de recetas manipuladas de fármacos psicotrópicos: implicaciones para el uso racional de medicamentos

Talita Garcia Lopes Viçoso ${ }^{*}$, Fernanda Maria Pagane Guereschi Ernandes ${ }^{1}$, Mariane Daniella da Silva ${ }^{2}$, Letícia Biazzi de Lima ${ }^{1}$, Jaciara Jesus Silva ${ }^{1}$, Jesley Pires Tomaz Silva1', Maria Laura Ferreira Martins ${ }^{1}$, Lya Bueno de Carvalho1.

\section{RESUMO}

Objetivo: Verificar a execução da legislação à prescrição e dispensação dos medicamentos psicotrópicos das listas B e C, definidas pela Portaria no 344/98 - SVS/MS. Métodos: Realizou-se uma pesquisa básicoaplicada, de abordagem quali-quantitativa. Foram obtidas informações de 570 prescrições manipuladas em uma farmácia de São José do Rio Preto - SP, entre setembro de 2018 a março de 2019. Foram analisadas as variáveis: legibilidade dos dados; identificação do emitente e usuário; nome do medicamento; quantidade e forma farmacêutica, dosagem e posologia; quantidade aviada; data de emissão; prazo de dispensação; assinatura; especialidade do prescritor; cumprimento do médico e/ou da farmácia no preenchimento dos campos pelos quais são responsáveis. Foi utilizado o software IBM SPSS Statistics. O estudo foi aprovado por Comitê de Ética em Pesquisa. Resultados: Foi observado que a maior porcentagem de falhas no preenchimento foi cometida pelos médicos em relação à farmácia e, entre as principais causas de erros, destacou-se a ilegibilidade. Conclusão: Apesar de uma legislação específica e clara, verifica-se a necessidade de conscientização por parte dos profissionais sobre o conhecimento da realização de uma prescrição de medicamentos psicotrópicos, assim como de sua dispensação, para garantia da terapêutica segura e eficaz ao usuário.

Palavras-chave: Psicotrópicos, Legislação, Prescrição, Benzodiazepínicos, Atenção farmacêutica.

\begin{abstract}
Objective: To assess compliance with the legislation regarding the prescription and dispensing of psychotropic drugs in lists B and C, defined by Ordinance 344/98 - SVS / MS. Methods: A basic-applied research with a quali-quantitative approach was carried out. The information was obtained from 570 prescriptions manipulated in a pharmacy in São José do Rio Preto - SP, between September 2018 and March 2019. Were analyzed: data legibility; issuer and user identification; drug name; quantity and pharmaceutical form, dosage; quantity filled; date of issue; dispensation term; signature; specialty of the prescriber and compliance with the doctor and/or pharmacy in completing the fields for which they are responsible. For data analysis, the IBM SPSS Statistics software was used. The study was approved by the Research Ethics Committee. Results: It was observed that the highest percentage of errors in filling was made by doctors in relation to the pharmacy and, among the main causes of errors, illegibility was highlighted. Conclusion: Despite specific and clear legislation, there is a need for awareness on the part of professionals about the knowledge of carrying out a prescription for psychotropic drugs, as well as their dispensation, to ensure safe and effective therapy for the user.
\end{abstract}

Keywords: Psychotropics, Legislation, Prescription, Benzodiazepines, Pharmaceutical attention.

1 Universidade Paulista (UNIP), São José do Rio Preto - SP. *E-mail: talita@vicoso.com.br

2 Universidade Estadual Paulista “Júlio de Mesquita Filho" (UNESP), São José do Rio Preto - SP. 


\section{RESUMEN}

Objetivo: Evaluar el cumplimiento de la legislación relativa a la prescripción y dispensación de drogas psicotrópicas de las listas B y C, definida por la Ordenanza No 344/98 - SVS/MS. Métodos: Se llevó a cabo una investigación básica aplicada, con un enfoque cuantitativo. La información se obtuvo de 570 recetas manejadas en una farmacia en São José do Rio Preto - SP, entre septiembre de 2018 y marzo de 2019. Se analizaron las siguientes variables: legibilidad de los datos; identificación del emisor y del usuario; nombre del medicamento; cantidad y forma farmacéutica, dosis y dosificación; cantidad aviada; fecha de emisión; período de dispensación; firma; especialidad del prescriptor; cumplimiento del médico y/o farmacia en la realización de los campos de los que son responsables. Se utilizó el software Ibm SPSS Statistics. El estudio fue aprobado por el Comité de ética de la investigación. Resultados: Se observó que el mayor porcentaje de fallos en el llenado fue cometido por los médicos en relación con la farmacia y, entre las principales causas de errores, se destacó la ilegibilidad. Conclusión: A pesar de una legislación específica y clara, hay una necesidad de concienciación por parte de los profesionales sobre el conocimiento del rendimiento de una receta de drogas psicotrópicas, para garantizar una terapia segura y eficaz al usuario.

Palabras-clave: Psicotrópicos, Legislación, Prescripción, Benzodiazepinas, Atención farmacéutica.

\section{INTRODUÇÃO}

A preocupação com problemas de saúde relacionados a medicamentos tem aumentado entre os profissionais da saúde. Vários são os fatores que se entrelaçam para que se consiga o uso racional do medicamento, entendido como o processo que compreende a prescrição apropriada, a disponibilidade oportuna e a preços acessíveis, a dispensação em condições adequadas, o consumo nas doses e pelo período de tempo indicados e nos intervalos definidos de medicamentos eficazes, seguros e de qualidade (MATTA SR, et al., 2011).

Um estudo realizado por Pelegrini MRF (2003) relata a preocupação com o aumento abusivo no uso de medicamentos psicotrópicos na atualidade. A depressão, a ansiedade e outros transtornos são fatores que contribuem cada vez mais para esse aumento, o que agrava um grande problema social: a automedicação. O ser humano está em constante busca pela felicidade, quer aliviar as dores e alcançar o prazer mesmo que seja de forma artificial, mas, ao ignorar que esses medicamentos podem causar dependência química e psíquica, coloca em risco a própria saúde.

O uso de medicamentos psicotrópicos ou psicoativos tem sido relacionado às causas de intoxicação humana. Segundo o Sistema Nacional de Informações - Tóxico-Farmacológicas (SINITOX), 30,7\% dos casos de intoxicação humana registrados, em 2008, no Brasil, tiveram os medicamentos como agentes tóxicos (SINITOX, 2018). Em 2006, a Associação Americana de Centros de Controle de Intoxicação (AAPCC) relatou que a categoria formada por sedativos, hipnóticos e antipsicóticos ocupou o quarto lugar entre as 25 categorias de agentes químicos mais frequentemente envolvidos com intoxicações humanas nos Estados Unidos (COSTA CSC, et al., 2019).

Dadas as particularidades do uso de medicamentos psicoativos, algumas medidas ensejam promover seu uso racional e, com isso, diminuir os riscos, frente aos possíveis benefícios da terapia. Entre elas estão as de cunho regulador, especialmente aplicáveis aos medicamentos psicoativos. No Brasil, essas medidas estão presentes na legislação sanitária vigente (BRASIL, 1973; BRASIL, 1999), que inclui normas relativas a produção, importação e comércio, prescrição, dispensação e uso de psicoativos. As normativas produzidas por Conselhos de classe - entre eles os de Medicina e Farmácia - também dispõem sobre a prescrição e dispensação desses medicamentos (CONSELHO FEDERAL DE MEDICINA, 1997; CONSELHO FEDERAL DE FARMÁCIA, 2004) e fiscalizam o exercício profissional daqueles que os prescrevem e dispensam (BRASIL, 1960).

A RDC 67, de 8 outubro de 2007, dispõe sobre Boas Práticas de Manipulação de Preparações Magistrais e Oficinais para Uso Humano em Farmácias e tem como objetivo fixar os requisitos exigidos para o exercício das atividades de manipulação das farmácias. Além de todo controle na aquisição da matéria-prima e manipulação do produto, destaca a importância da Atenção Farmacêutica aos usuários ou seus responsáveis, visando à segurança do paciente para o uso seguro e racional de medicamentos. 
Ainda que o rigor das normas tenha como intuito auxiliar o controle do uso e do comércio dessas substâncias, é possível que esse sistema normativo seja inefetivo, não apenas no controle da utilização, mas também na promoção do uso racional. Essa situação poderia ser primeiramente verificada pela consistência interna do próprio sistema, isto é, pela complementaridade ou conflito entre as normas (NOTO AR, et al., 2002; ANDRADE MF, et al., 2004). Outra questão é a adequada formação dos profissionais para desempenho das atividades de prescrição e de dispensação de medicamentos, que contribui para o sucesso das medidas reguladoras (SILVA LRM e VIEIRA EM, 2004).

A maioria dos problemas de origem psicológica ou psicossocial é vista pelo clínico geral, no atendimento primário. Se a prescrição de psicotrópicos iniciada neste momento for errônea, pode ter início um círculo vicioso que muitas vezes dura por vários anos. Nesse sentido, este estudo irá descrever e analisar, do ponto de vista de sua contribuição para o uso racional de medicamentos, os instrumentos legais pelos quais se realiza a regulação quanto à prescrição médica e à dispensação de medicamentos no Brasil, especialmente os psicoativos, dentre os medicamentos sujeitos a controle especial.

\section{MÉTODOS}

Em relação à natureza, esta pode ser considerada uma pesquisa básico-aplicada, com intuito de levar informações à área médica sobre o assunto a ser estudado. Quanto ao método, a abordagem foi classificada como quali-quantitativa, por meio da qual realizaram-se análises de dados e suas quantidades.

Para a determinação do universo da pesquisa e planejamento das amostras, foram obtidas informações de prescrições de pacientes retidas em farmácia de manipulação do município de São José do Rio Preto-SP, referentes ao período entre setembro de 2018 e março de 2019.

Os procedimentos de pesquisa foram o experimental e o bibliográfico, sendo consultados livros, dissertações e teses. Para análise dos dados utilizou-se o software IBM SPSS Statistics. O estudo foi aprovado por Comitê de Ética em Pesquisa sob o número 3.065.147.

Dentre as 570 prescrições escolhidas, 274 referem-se a prescrições da Lista B e 296, da Lista C1. Para avaliar o cumprimento da legislação foram analisados os seguintes itens: 1- Legibilidade das informações preenchidas de forma manuscrita. 2- Identificação do médico ou da instituição constando nome, endereço e CRM. 3- Identificação do paciente: nome e endereço completos. 4- Nome do medicamento ou da substância, de acordo com a Denominação Comum Brasileira (DCB). 5- Dosagem do remédio escrita em números arábicos e a posologia. 6- Quantidade aviada. 7- Data de emissão. 8- Verificação do prazo (30 dias). 9Assinatura do médico com o CRM e carimbo. 10- Especialidade de quem prescreveu o medicamento. 11Cumprimento do médico e/ou da farmácia no preenchimento dos campos pelos quais são responsáveis.

Foi adotada como critério para considerar falha a ocorrência de ao menos um campo não preenchido para prescrições $\mathrm{B}$ e $\mathrm{C} 1$. Para as receitas $\mathrm{B}$, os critérios de análise foram: 1- Identificação da Unidade da Federação e o número da receita. 2- Identificação do paciente ou comprador: nome, endereço, identidade e telefone. 3Identificação da farmácia: nome, endereço e identificação do responsável pela venda. 4- Gráfica: nome, endereço, CGC, número da autorização da ANVISA, número inicial e final. Para receitas C1: 1- Lançamento da informação sobre registro no livro.

\section{RESULTADOS E DISCUSSÃO}

A prescrição de medicamentos é um documento com valor legal pelo qual se responsabilizam, perante o paciente e a sociedade, aqueles que prescrevem, manipulam, dispensam e administram os medicamentos. Ela deve conter informações claras que instruam os pacientes e demais profissionais de saúde, garantindo a fidelidade da interpretação e a objetividade da informação (CASTRO CGSO e PEPE VLE, 2012; VALADÃO AF, et al., 2019).

Entre muitos fatores que contribuem para o uso racional de medicamentos, está a prescrição adequada. A prescrição racional deve basear-se em várias etapas: diagnóstico específico, implicações fisiopatológicas, objetivo terapêutico para cada processo fisiopatológico, posologia adequada, monitoramento da ação farmacológica e determinação do término do tratamento. Todos os fatores são importantes para se alcançar 
o uso racional dos medicamentos, incluindo a análise do farmacêutico sobre a prescrição do medicamento, que, deve examinar a prescrição e dispensar o medicamento caso este não apresentar irregularidades no receituário, além de incluir orientações ao paciente contribuindo para o uso racional de medicamentos (ALVES TNP, et al., 2012).

Deste modo, foi avaliada a verificação da legislação quanto a prescrição e venda dos medicamentos psicotrópicos das listas B e C1 presentes na Portaria no $344 / 98$ - SVS/MS. Todas as prescrições pertencentes à lista $B(274)$ manipuladas pelo estabelecimento participante, durante o período de setembro de 2018 a março de 2019, foram avaliadas, já as prescrições da lista C1 (296) tiveram como critério de análise a forma aleatória, pois a quantidade de prescrições de medicamentos da lista $C 1$ é maior que lista $B$ e o estudo têm como critério avaliar quantidades equivalentes das listas $\mathrm{B}$ e $\mathrm{C} 1$. As visitas à farmácia foram realizadas semanalmente e acompanhadas pelo farmacêutico responsável do estabelecimento, que prestou esclarecimento sobre todos os questionamentos levantados. Os resultados foram abordados de acordo com o cumprimento dos critérios exigidos pela legislação.

Todos os itens avaliados neste trabalho merecem destaque para garantir o sucesso no tratamento e proteger o paciente de possíveis erros na prescrição, que podem ser fatais. Apesar de uma legislação específica e clara, ainda se encontram muitos erros nas prescrições medicamentosas. Entre as principais causas de erros, destaca-se a ilegibilidade. Segundo Aguiar G, et al. (2006), as prescrições devem ser legíveis, apresentarem informações claras e completas para que o farmacêutico possa desempenhar sua função de dispensação e orientação corretamente. Este estudo mostrou que, das 570 prescrições analisadas entre as listas B e C1, 63,5\% estavam ilegíveis. A ilegibilidade é um fator muito preocupante, porque medicamentos com nomes similares podem ser dispensados incorretamente (ANACLETO TA, et al., 2010).

De acordo com a Portaria investigada, é obrigatório que constem as informações de identificação do emitente, ou seja, formulário do médico ou da instituição apresentando o nome, o endereço completo e o número do CRM, pois, diante da necessidade de qualquer esclarecimento sobre a prescrição, é necessária a comunicação com o prescritor. Os resultados apresentados neste trabalho referentes a esse item mostram que os prescritores cumpriram a legislação, pois apenas uma prescrição $(0,34 \%)$ referente à lista de medicamentos $\mathrm{C} 1$ não apresentou identificação do emitente, ao passo que todas das receitas da lista $B$ mostraram essa informação. Vale ressaltar que em algumas receitas não se constou a especialidade médica, mas nesse tópico somente foi analisada a identificação do emitente, sendo que a lei exige o formulário do médico ou da instituição constando: nome, endereço e CRM.

Entretanto, quando se avaliou a identificação do usuário, não se obteve o mesmo resultado quando comparado com a identificação médica, visto serem percebidas falhas no preenchimento dos dados do paciente. Das prescrições analisadas, $38,6 \%$ estavam incompletas, pois não possuíam o endereço completo do usuário (107 da lista C1 e 113 da lista B).

Quanto à prescrição do nome do medicamento de acordo com a DCB (Denominação Comum Brasileira), exigida pelo Ministério da Saúde, este estudo, quando comparado a outros estudos, traz um resultado diferente. Segundo Andrade MF, et al. (2004), das 753 prescrições avaliadas em seu estudo, 78,2\% das prescrições da lista $C 1$ e 53,1\% da lista B não estavam de acordo com a DBC, já este trabalho mostra uma outra realidade de resultados, pois $100 \%$ das prescrições cumpriram a legislação que determina a descrição do medicamento de acordo com a DCB.

O Gráfico 1 mostra a frequência (\%) de prescrição/dispensação de substâncias sujeitas a controle especial da lista C1 da Portaria $n^{\circ} 344 / 98$ - SVS/MS. Os medicamentos mais prescritos foram a nortriptilina $(25,68 \%)$ e em seguida amitriptilina (18,24\%), ambos medicamentos antidepressivos tricíclicos. Esses medicamentos são modificadores seletivos do Sistema Nervoso Central. Atuam na recaptação da norepinefrina e serotonina no SNC com propriedades estimulantes e depressoras ao mesmo tempo. A sua indicação é para aliviar sintomas de depressão.

A depressão é diagnosticada a partir de uma entrevista clínica e traz como principais características: humor deprimido, déficits na cognição, distúrbios do sono e apetite e perda de energia. O paciente ou o responsável deve ser orientado sobre os benefícios e riscos causados pelo medicamento, afinal o medicamento em 
questão possui muitos efeitos colaterais e também interações medicamentosas. Não deve ser utilizado por crianças, somente para adultos, que não devem consumir bebidas alcoólicas. Portanto, medicamentos psicotrópicos merecem toda a atenção e cuidado no momento da prescrição, dispensação e acompanhamento farmacoterapêutico.

O uso de medicamentos psicotrópicos é crescente, pois os distúrbios de natureza psíquica estão cada vez mais afetando a população, motivo pelo qual se fizeram necessários o controle e a fiscalização dessas substâncias (TORRES MLD, et al., 2014).

Gráfico 1 - Frequência de prescrição/dispensação de substâncias sujeitas a controle especial da lista C1 da Portaria n 344/98 - SVS/MS, n.296. São José do Rio Preto-SP. 2019.

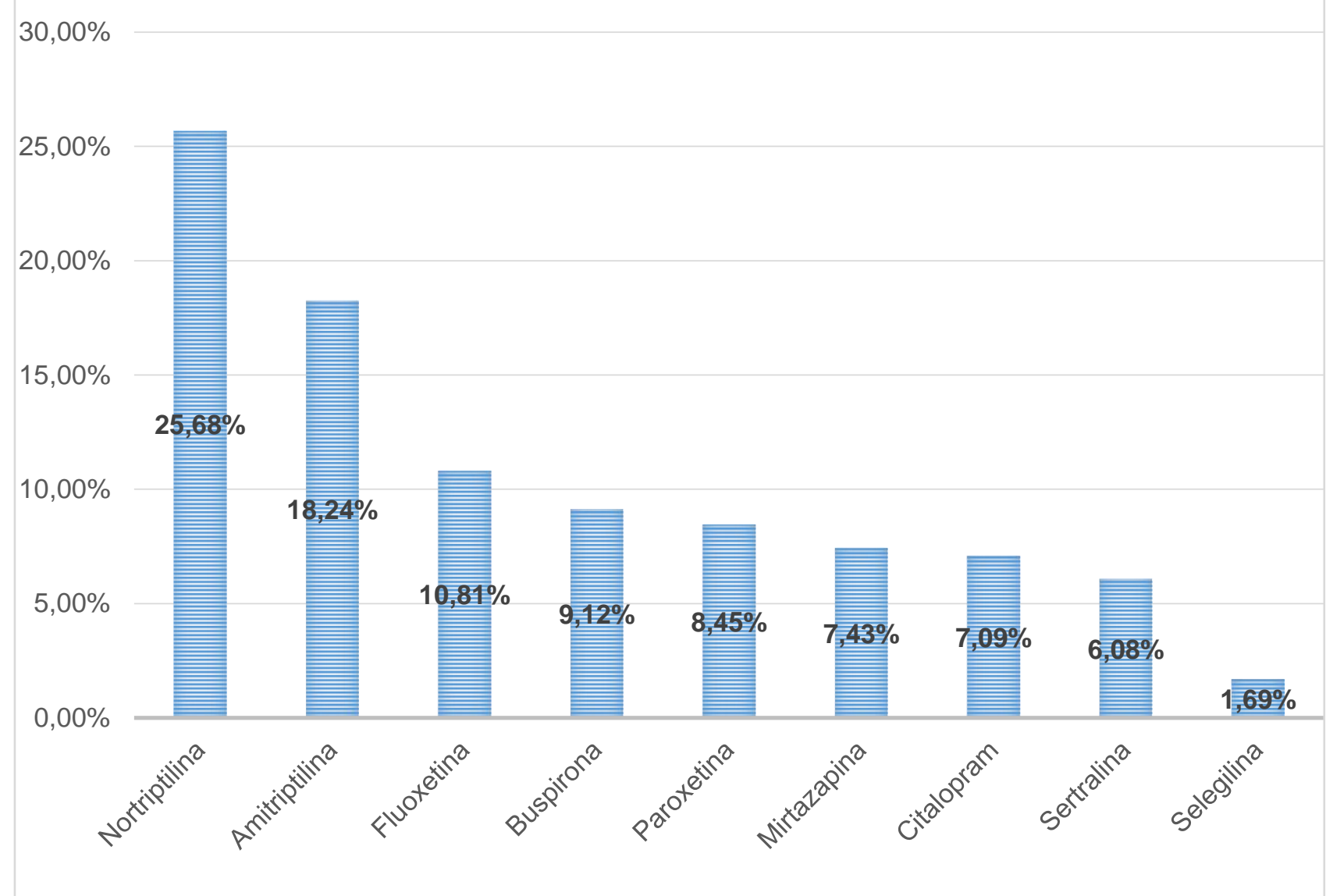

Fonte: Viçoso TGL, et al., 2020.

O Gráfico 2 representa a frequência (\%) de prescrição/dispensação de substâncias sujeitas a controle especial da lista B1 da Portaria $n^{\circ} 344 / 98$ - SVS/MS. O psicotrópico predominantemente mais prescrito da lista B foi o benzodiazepínico Clonazepam (22,99\%). Um estudo de Torres MLD, et al. (2014) concorda com esse resultado, indicando o Clonazepam como medicamento mais dispensado em seu trabalho; $33,82 \%$ das prescrições aviadas foram do medicamento citado; o mesmo autor aponta um estudo de Firmo, WCA, et al. (2013) com o mesmo resultado.

Segundo a bula do medicamento, o Clonazepam é um medicamento indicado para: distúrbio epiléptico; transtornos de ansiedade; transtornos do humor; emprego em síndromes psicóticas; tratamento da síndrome das pernas inquietas; tratamento da vertigem e sintomas relacionados à perturbação do equilíbrio; e tratamento da síndrome da boca ardente. Ele pode ser administrado com outros antiepilépticos, mas sob monitoramento e avaliação cuidadosa e sua posologia é estabelecida de acordo com a indicação clínica e de forma individual. Como todo sedativo-hipnótico, ele também tem interações farmacodinâmicas e farmacocinéticas e não deve ser administrado com álcool. 
Gráfico 2 - Frequência (\%) de prescrição/dispensação de substâncias sujeitas a controle especial da lista B1 da Portaria n 344/98 - SVS/MS, n.274. São José do Rio Preto-SP. 2019.

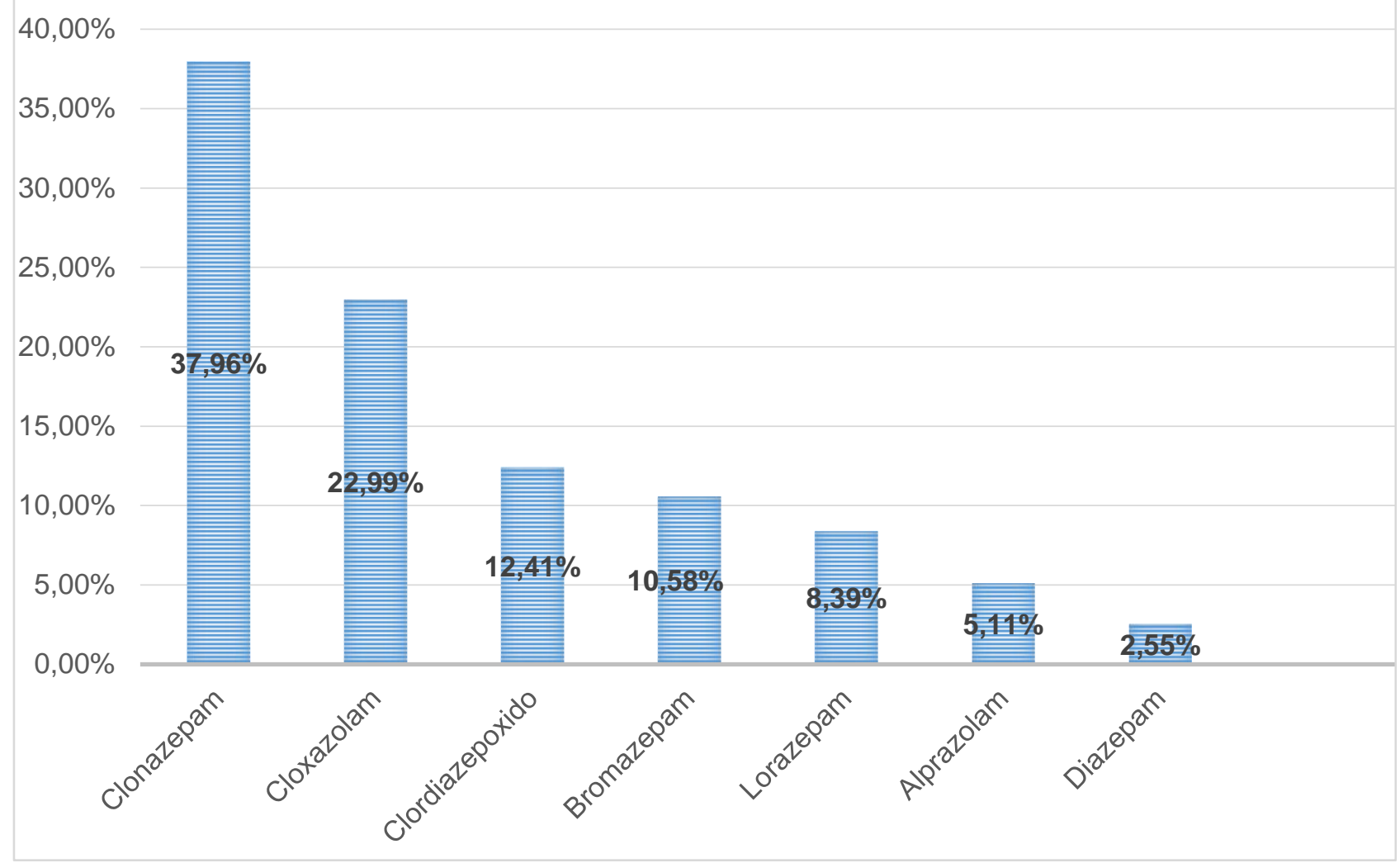

Fonte: Viçoso TGL, et al., 2020.

Entre muitos fatores que contribuem para o uso racional de medicamentos, está a prescrição adequada (SILVÉRIO MS e LEITE ICG, 2010). A prescrição racional deve basear-se em várias etapas: diagnóstico específico, implicações fisiopatológicas, objetivo terapêutico para cada processo fisiopatológico, posologia adequada, monitoramento da ação farmacológica e determinação do término do tratamento. Sobre a porcentagem de erros envolvendo médicos e farmácias no preenchimento de todos os campos do medicamento sujeito a controle especial, da lista B e C1 da Portaria $n^{\circ} 344 / 98$ - SVS/MS pelos quais são responsáveis, a maior porcentagem de falha observada foi referente à prescrição médica, tanto para a lista $B$ $(97,81 \%)$, como para C1 $(95,95 \%)$, enquanto as falhas observadas pela farmácia apresentaram-se na lista B $(46,35 \%)$.

A legislação exige que a quantidade seja descrita em arábico e extenso, que contenha forma farmacêutica e posologia, mas na análise das prescrições foram encontradas falhas no preenchimento correto. As prescrições continham as informações da quantidade de medicamento em arábico, forma farmacêutica e posologia corretamente, mas $93,16 \%$ não apresentaram o valor do medicamento escrito por extenso. Foram encontradas prescrições sem data e em quantidades superiores permitidas por lei (60 dias), o que compromete o acompanhamento correto do paciente que faz uso do medicamento, fato que pode contribuir para ocorrência de erros de medicação.

É importante considerar que são substâncias psicotrópicas que afetam o humor e o comportamento dos seres humanos, afinal agem nos neurotransmissores centrais com implicações sistêmicas, portanto quando utilizados de forma abusiva podem causar graves danos ao paciente, comprometendo sua saúde, além de causar dependência e interação medicamentosa (NASARIO M e SILVA MM, 2016).

Todos são fatores de suma importância para se alcançar o uso racional de medicamentos, mas, não se pode esquecer a fundamental importância do farmacêutico que deve analisar a prescrição e dispensar o medicamento quando este não apresentar irregularidades no receituário, além de incluir orientações ao paciente (ALVES TNP, et al., 2012). 
As falhas que envolveram a farmácia foram de $46,35 \%$ para medicamentos da lista B e $0 \%$ para lista $C 1$ e estas eram mais relacionadas à falta de identificação do comprador do que à identificação da farmácia, itens obrigatoriamente avaliados nas receitas da lista B. A farmácia participante da pesquisa cumpriu corretamente a legislação em relação ao item de lançamento da informação sobre registro no livro; todas as prescrições referentes à lista $\mathrm{C} 1$ foram lançadas e o responsável pelo estabelecimento relatou que há um controle de compra e venda sobre as substâncias relacionadas a medicamentos psicotrópicos, tudo é repassado ao Sistema Nacional de Gerenciamento de Produtos Controlados (SNGPC) e Vigilância Sanitária.

A resolução de 30 de março de 2007 dispõe sobre o SNGPC, estabelecendo normas de implantação para drogarias e farmácias (BRASIL, 2007); o SNGPC tem por objetivos: monitorar a dispensação de medicamentos e substâncias entorpecentes e psicotrópicas e seus precursores; otimizar o processo de escrituração; permitir o monitoramento de hábitos de prescrição e consumo de substâncias controladas em determinada região, para propor políticas de controle; captar dados que permitam a geração de informação atualizada e fidedigna para o Serviço Nacional de Vigilância Sanitária (SNVS), para a tomada de decisão; dinamizar as ações da vigilância sanitária (BRASIL, 2010). De acordo com a dispensação no prazo previsto segundo a legislação, o prazo correto para a dispensação é de 30 dias após a prescrição.

Nas prescrições da lista C1, 91,89\% (272 prescrições) das receitas estavam dentro do prazo estipulado e da B, 95,99\% (263 prescrições). Ao avaliar o número da receita, a identificação da Unidade de Federação e os dados da gráfica, como o nome, endereço, CGC e o número de autorização da ANVISA, as receitas estavam todas dentro das normas. A identificação da farmácia estava presente em 95,62\%, ou seja, 262 das prescrições avaliadas. Quanto à identificação correta do comprador, $55,47 \%$ das receitas analisadas apresentavam todos os campos do comprador preenchidos corretamente. $O$ item correspondente ao lançamento da informação sobre registro do livro foi realizado somente para receitas $\mathrm{C} 1$ e observou-se que todas as prescrições foram lançadas no livro. Em relação à prescrição por especialidade médica, destacaramse quatro especialidades nas prescrições. Para os medicamentos da lista $\mathrm{C} 1$ foram: psiquiatras $(26,01 \%)$ tanto para a lista B quanto para a C1, em seguida, neurologistas (16,55\%), reumatologistas $(16,22 \%)$ e geriatras (13,85\%) (Gráfico 3).

Gráfico 3 - Prescrições por especialidade médica para medicamentos da lista C1, n.274. São José do Rio Preto-SP. 2019.

Psiquiatra
Neurologia
Reumatologia
Geriatra
Sem especialidade
Nutrologia
Outros

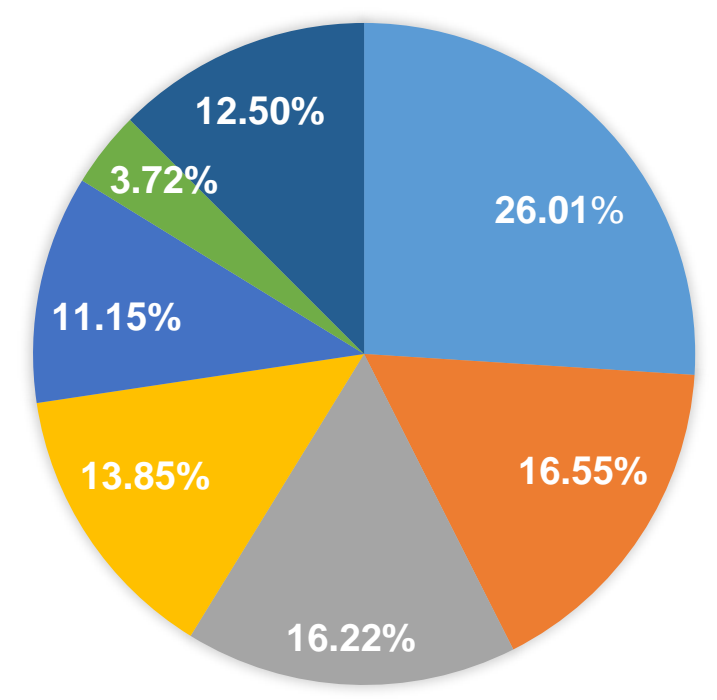

Fonte: Viçoso TGL, et al., 2020.

Na prescrição dos medicamentos da lista B, em primeiro lugar manteve-se a especialidade de psiquiatras (35,77\%) e, em seguida, os nutrólogos (21,53\%); os cirurgiões $(11,31 \%)$ e os neurologistas $(9,49 \%)$. Os dados relatados estão representados no Gráfico 4. 
Gráfico 4 - Prescrições por especialidade médica para medicamentos da lista B, n.274. São José do Rio Preto-SP. 2019.

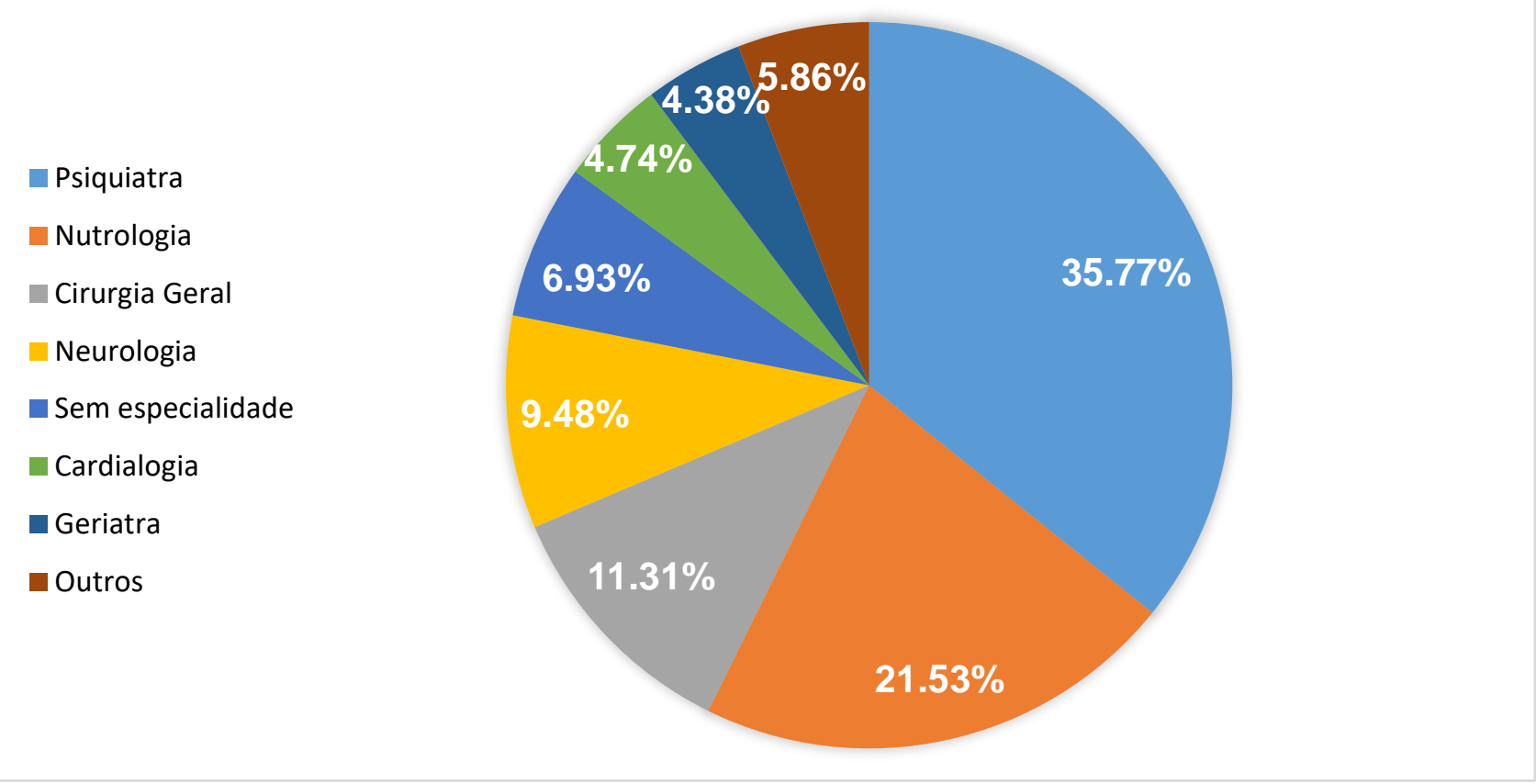

Fonte: Viçoso TGL, et al., 2020.

Diante dos dados obtidos no Gráfico 4, deve-se destacar o número de prescrições sem especialidade médica, na lista $C 1(11,15 \%)$ e na $B(6,93 \%)$, fato preocupante por se tratar de substâncias psicotrópicas. Segundo Ferrari CKB, et al. (2013), o correto seria que esse tipo de medicamento fosse prescrito por psiquiatras e neurologistas que são especialistas nesse tipo de medicação, pois muitas vezes essa classe de medicamentos é prescrita de forma equivocada, comprometendo a qualidade da prescrição e causando prejuízos à saúde do paciente.

\section{CONCLUSÃO}

As prescrições avaliadas mostraram que a maioria das prescrições médicas se encontra de acordo com o regulamento técnico dos medicamentos e substâncias com controle especial, descritos na Portaria no 344/98 - SVS/MS, porém, ainda foram encontradas muitas falhas que devem ser corrigidas por meio da sensibilização dos profissionais envolvidos com a prescrição. Cabe a eles buscar informações para se especializarem e cumprirem seu dever perante o compromisso assumido com sua profissão. A prescrição realizada de forma adequada é a primeira responsável para o processo de cura do paciente, seguida da etapa de dispensação e orientação realizada pelo profissional farmacêutico, a qualidade de uma prescrição irá garantir o uso racional de medicamentos pelo paciente e a eficácia no tratamento. A falha em uma das partes compromete a saúde do paciente, podendo causar danos irreparáveis.

\section{REFERÊNCIAS}

1. AGUIAR G, et al. llegibilidade e ausência de informação nas prescrições médicas: fatores de risco relacionados a erros de medicação. RBPS. 2006; 19(2): 84-91.

2. ALVES TNP, et al. Análise das prescrições médicas em unidades de atenção primária à saúde do município de Juiz de Fora - MG. Convibra. 2012.

3. ANACLETO TA, et al. Erros de medicação. Farmácia Hospitalar. Pharmacia Brasileira. 2010; 1(1-24).

4. ANDRADE MF, et al. Prescrição de psicotrópicos: avaliação das informações contidas em receitas e notificações. Revista Brasileira Ciências Farmacêuticas, 2004; 40(4): 471-479.

5. BRASIL. Lei Federal 9. Brasília: Diário Oficial da União, 10 de fevereiro de 1999.

6. BRASIL. Lei ํㅜ 3820. Brasília: Diário Oficial da União, 11 de novembro de 1960. 
7. BRASIL. Lei no 5991. Brasília: Diário Oficial da União, 17 de dezembro de 1973.

8. BRASIL. Ministério da Saúde. Agência Nacional de Vigilância Sanitária. Portaria SVS/MS no 344. Brasília: Diário Oficial da União, 12 de maio de 1998.

9. BRASIL. Ministério da Saúde. Agência Nacional de Vigilância Sanitária. Resolução RDC no. 27. Brasília: Diário Oficial da União, 30 de março de 2007.

10. BRASIL. Ministério da Saúde. Agência Nacional de Vigilância Sanitária. Sistema Nacional de Gerenciamento de Produtos Controlados - SNGPC. 2010.

11. BRASIL. Resolução - RDC MS/ANVISA №138. Brasília: Diário Oficial da União, 29 de maio de 2003.

12. CASTRO CGSO, PEPE VLE. Prescrição de medicamentos. Escola Nacional de Saúde Pública - Ministério da Saúde. 2012.

13. CONSELHO FEDERAL DE FARMÁCIA. Resolução no 308. Brasília: Diário Oficial da União, 2 de maio de 1997.

14. CONSELHO FEDERAL DE MEDICINA. Resolução CFM no 1.477. Brasília: Diário Oficial da União, 11 de julho de 1997.

15. COSTA CSC, et al. Atenção farmacêutica nas intoxicações por automedicação. Referências em Saúde, 2019; 2(1): 114-120.

16. FERRARI CKB, et al. Falhas na Prescrição e Dispensação de Medicamentos Psicotrópicos: Um problema de Saúde Pública. Rev Ciênc Farm Básica Apl., 2013;34(1): 109-116.

17. FIRMO WCA, et al. Análise das prescrições médicas de psicotrópicos de uma farmácia comercial no município de Bacabal, Maranhão. J Manag Prim Health Care, 2013; 1(4): 10-18.

18. MATTA SR, et al. Prescrição e dispensação de medicamentos psicoativos nos instrumentos normativos da regulação sanitária brasileira: implicações para o uso racional de medicamentos. Rev. Bras. Farm. 2011; 92(1): 33-41.

19. NASARIO M, SILVA MM. O Consumo excessivo de medicamentos psicotrópicos na atualidade. 2016.

20. NOTO AR, et al. Análise da prescrição e dispensação de medicamentos psicotrópicos em dois municípios do Estado de São Paulo. Revista Brasileira de Psiquiatria, 2002; 24(2): 68-73.

21. PELEGRINI MRF. O abuso de medicamentos psicotrópicos na contemporaneidade. Psicol. Cienc. Prof., 2003; 23(1).

22. SILVA LRD, VIEIRA EM. Conhecimento dos farmacêuticos sobre legislação sanitária e regulamentação da profissão. Revista de Saúde Pública, 2004; 38: 429-437.

23. SILVÉRIO MS, LEITE ICG. Qualidade das prescrições em município de Minas Gerais: uma abordagem farmacoepidemiológica. Rev Assoc Med Bras, 2010; 56(6): 675-80.

24. SINITOX - Sistema Nacional de Informações Tóxico-farmacológicas. 2018.

25. SOUSA LMG, et al. Estudo de prescrições médicas de psicotrópicos de uma farmácia comercial no município de Santa Inês, Maranhão, Brasil. Centro Cientifico Conhecer, 01 dez. 2014.

26. TORRES MLD, et al. Prescrição de psicotrópicos e especialidades médica: estudo em uma farmácia comercial no município do Maranhão. Revista científica do ITPAC, 2014; 7(4).

27. VALADÃO AF, et al. Prescrição médica: um foco nos erros de prescrição. Rev Bras Farm., 2009; 90(4): $340-343$. 